\title{
Indigenous marine diatoms as novel sources of bioactive peptides with antihypertensive and antioxidant properties
}

\begin{abstract}
Several bioactive compounds from microalgae have demonstrated diverse biological activities with positive effects on human health. However, the potential of bioactive peptides as functional foods is still undervalued. Therefore, the exploration of microalgae strains as sources of bioactive peptides could reveal strong and unique bioactivities, especially when these marine sources have never been explored before. For this aim, protein extracts from six indigenous marine diatoms were subjected to enzymatic hydrolysis using four proteases (flavourzyme, pepsin, papain and trypsin). The hydrolysates were then tested for angiotensin converting enzyme (ACE)-inhibitory, antioxidant and antihypertensive properties. Results showed that papain hydrolysates from all microalgae strains exhibited strong ACE-inhibitory activities and antioxidant properties. In particular, protein hydrolysates from Bellerochea malleus were found to reduce blood pressure properties of $17 \mathrm{mmHg}$ after 5 days of oral administration to SHR animals. These results revealed the potential of bioactive peptides from indigenous marine diatoms for use as functional foods or nutraceuticals.
\end{abstract}

Keyword: ACE-inhibitory activity; Antihypertensive activity; Antioxidant activity; Marine microalgae; Protein hydrolysates 